\title{
Microprocessor System for Thermal Food Process Monitoring Based on Zigbee Technology
}

\author{
Krassimir Kolev ${ }^{1}$ \\ ${ }^{1}$ University of Food Technologies, Department of Computer Systems and Technologies, \\ 26 Maritza Bld., Plovdiv 4000, Bulgaria, European Union
}

\begin{abstract}
This paper presents a novel microprocessor system for thermal food process monitoring based on new wireless technology. The paper presents a low-cost and highly versatile temperature-monitoring system applicable to all phases of thermal food processing. The design diagram of the microprocessor system is synthesized. The operating principle of the microprocessor system is explained. The microprocessor system for thermal food process monitoring is implemented by new embedded microcontroller CC2430 with $x 51$ core of the Texas Instruments. The schematic diagram is given. An algorithm diagram on the microprocessor system for thermal food process monitoring is synthesized. Analyses of the proposed microprocessor system are made. The system has been tested under laboratory conditions and in real-world operational applications. Recommendations for maintenance are given.
\end{abstract}

Keywords: microprocessor system, food processing, thermal monitoring, Zigbee.

\section{Introduction}

Thermal processes are essential in the preservation of food products. The process of pasteurization and sterilization are among the most responsible in food processing requiring permanent temperature monitoring of the product in package [2]. For determining the degree of heat treatment is necessary to take into account both the change of the temperature of the food and the time of thermal effect on the product. There are different options for the heat treatment depending on the food products. To determine the degree of heat treatment are used thermal integrated criteria. In case of sterilization common criteria for thermal effect is Lethality values

$$
F_{0}=\int_{0}^{t} 10^{\frac{(T-121.1)}{10}} d t,
$$

where the internal product temperature is $\mathrm{T}$ in ${ }^{\circ} \mathrm{C}$. For products with a $\mathrm{pH}$ less than 4.5 , so-called acid products, food poisoning organisms of the type Clostridium botulinum do not germinate; consequently it is only necessary to inactivate molds and yeasts. This can be done at much lower temperatures, with the result that the $\mathrm{F}_{0}$-values are very low, since the lethal rate at a temperature of $80^{\circ} \mathrm{C}$ is $7.76 \times 10^{-5}$ $\min ^{-1}$. A more practical unit for quantifying the lethal effect of this type of process is the pasteurization unit $\mathrm{P}$ given by

$$
P=\int_{0}^{t} 10^{\frac{T-65}{10}} d t,
$$

where the internal product temperature is $\mathrm{T}$ in ${ }^{\circ} \mathrm{C}$

In sterilization and pasteurization, it is necessary to know the temperature distribution obtained at the point of slowest heating in the food product during the process to calculate criterions for thermal effect.

Heat treatment recommendations with pasteurization units $P$ for some pasteurized products and Lethality values $\left(\mathrm{F}_{0}\right)$ for commercial sterilization of selected canned food products sterilized product are given in specialized literatures for food processing [1].
This design allows realizing the remote monitoring of the process of sterilization and pasteurization using the capabilities of the Zigbee technology for the realization of a wireless networks. Wireless sensor networks (WSNs) are more and more frequently seen as a solution to large-scale tracking and monitoring applications, because of their lowdata-rate, low-energy-consumption, and short-range link network which provides an opportunity to monitor and control the physical world to a previously unprecedented scale and resolution. Wireless sensor network (WSN) is a group of specialized autonomous sensors and actuators with a wireless communications infrastructure, intended to monitor and control physical or environmental conditions at diverse locations and to cooperatively pass their data to a main location and/or pass their control command to a desired actuator through the network. This paper is unique in bringing together wireless communication with new embedded microcontrollers for actual WSN design in field of food process technologies. The design integrates positives of new ZigBee technology with advances of novel true Systemon-Chip Solution (SoS) on embedded microcontrollers for 2.4-GHz IEEE 802.15.4 WSN realization.

\section{Design Diagram and Operating Principle}

\subsection{Requirements and design diagram}

Requirements to parameters of system for thermal food process monitoring are:

- the system must have both local and remote monitoring of thermal process;

- the system must calculate the integral F or P value;

- the system must have the capability of local control;

- local monitoring via LCD display;

- wireless connection to control center;

- the system must operate so that it allows to record data;

- low consumption for option for battery power supply.

Figure 1 shows the block diagram of the proposed microprocessor system. 


\section{International Journal of Science and Research (IJSR) \\ ISSN (Online): 2319-7064}

Index Copernicus Value (2013): 6.14 | Impact Factor (2014): 5.611

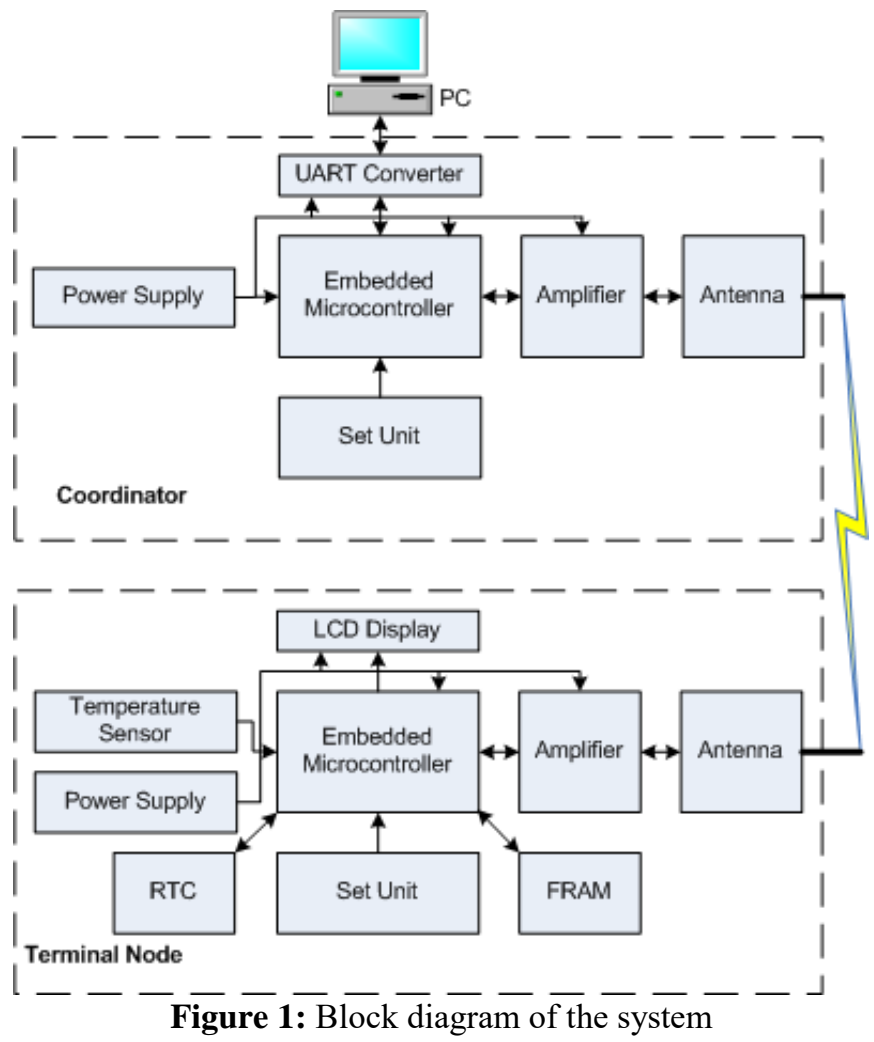

Terminal node parts of the microprocessor system are located close to the thermal processes while the coordinator part is located remotely. Coordinator part of the microprocessor system is just one, while the number terminal nodes are equal to the number of thermal monitoring objects. The microprocessor system in Figure 1 consists of:

- power supply which provides safe continuous voltage with battery back up for the operation of the system;

- LCD display for local monitoring;

- temperature probe to sense the thermal process temperature;

- set unit for selecting the operation mode;

- Embedded microcontroller with implanted Zigbee technology, memories and peripherals;

- FRAM is new fast write non-volatile memory with resistance to radiation and electromagnetic fields, and write endurance more than $10^{15}$ cycles;

- real-time clock with calendar (RTC) as time logger of the food processing.

- amplifier for signal booster;

- antenna which converts electric power into radio waves;

- UART converter interfaces the microprocessor system to host personal computer (PC).

\subsection{Operating principle and Zigbee communication}

The IEEE 802.15.4 standard defined in 2003 [3] was created for low-power devices that operate in the $868 \mathrm{MHz}, 915$ $\mathrm{MHz}$, and $2.45 \mathrm{GHz}$ frequency bands. Before this standard was developed, the ZigBee Alliance worked on a low-cost communication technology for low data rates and low power consumption. The IEEE and the ZigBee Alliance ultimately joined forces and ZigBee has become the commercial name for the IEEE 802.15.4 technology. One of the differences of the IEEE 802.15.4 standard is that it defines two types of node in the network. The two types of node are referred to as full-function devices (FFDs) and reduced-function devices (RFDs).An FFD is similar to a Bluetooth device and it can serve as the coordinator or master of a piconet or as a common node or a full-function slave. An FFD can communicate with any other device and it can help routing messages throughout the network. The RFD nodes are defined to be extremely simple with very modest resource and communication capabilities, only used as a slave node to communicate with an FFD. This provides flexibility for implementation of a variety of topologies addressing more diversified applications. A typical example application would be a wireless light lamp switch. The node at the lamp can be an FFD, since it is connected to the mains supply, while the battery powered light switch would be an RFD. IEEE 802.15.4 supports star, tree, cluster tree, and mesh networks. Figure 2 shows the star and peer-to-peer topologies of IEEE 802.15.4.
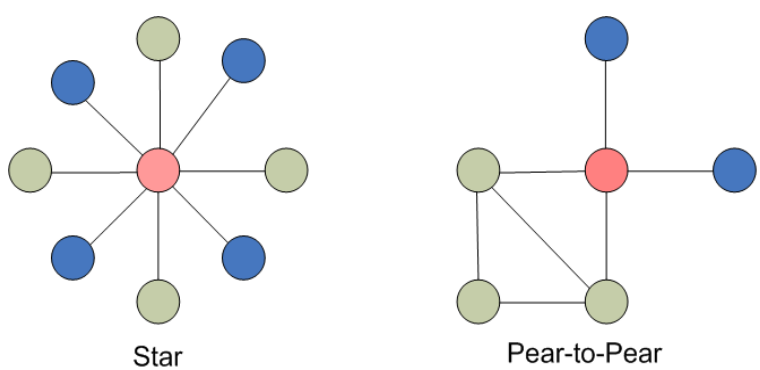

Pan Coordinato

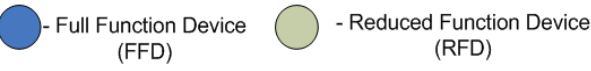

Figure 2: Star and peer-to-peer topologies of the Zigbee

The star topology is used to form star and tree networks, and the peer-to-peer topology to form cluster tree and mesh networks. In the star topology, a FFD serving as a coordinator is specified to be the central device, which is called the PAN coordinator, and starts and manage the whole network. Other coordinators and network devices must join the network by associating themselves with the PAN coordinator. The PAN coordinator controls all network communications. The peer-to-peer topology also requires a PAN coordinator to initialize the network start-up procedure. However, the communications within a network are based on the peer-to-peer topology and are not limited by the PAN coordinator. Any FFD device can freely talk to any other FFD device so long as they are within effective communication range. Any RFD device can talk only to its parent FFD device and cannot directly talk to any other RFD device. RFD devices and their parent FFD device form a tree topology. Cluster tree topology can be a single cluster network or a multi-cluster network.

The star topology for this thermal food process monitoring design is used because of its simple and reliable connection to coordinator. The Zigbee is very similar to others as wireless technology. Many applications and the coverage for ZigBee are similar to those of the Bluetooth. ZigBee provides data integrity check and Authentication. It uses collision avoidance mechanism, and at the same time it reserves a dedicated time slot to require a fixed bandwidth of the communication service. Transmitting and receiving

\section{Volume 4 Issue 12, December 2015}




\section{International Journal of Science and Research (IJSR) \\ ISSN (Online): 2319-7064 \\ Index Copernicus Value (2013): 6.14 | Impact Factor (2014): 5.611}

information has lower power consumption. ZigBee network can accommodate a maximum of 65536 devices. ZigBee network has self-organizing and self-healing capabilities. The performance comparison of Zigbee with other wide spread wireless technologies is given in table 1 .

Table 1: Performance comparison for widespread wireless technologies

\begin{tabular}{|c|c|c|c|}
\hline Features & ZigBee & Bluetooth & Wi-Fi \\
\hline $\begin{array}{c}\text { Working } \\
\text { frequency }\end{array}$ & $\begin{array}{c}2.4 \mathrm{GHz}, \\
868 / 915 \mathrm{MHz}\end{array}$ & $2.4 \mathrm{GHz}$ & $2.4 \mathrm{GHz}$ \\
\hline nodes per network & 65536 & 7 & 32 \\
\hline Wake-up time & $30 \mathrm{~ms}$ & $10 \mathrm{~s}$ & $3 \mathrm{~s}$ \\
\hline Data rate & $250 \mathrm{Kbps}$ & $1 \mathrm{Mbps}$ & $11 \mathrm{Mbps}$ \\
\hline Topology & star, tree, mesh & tree & tree \\
\hline Extension & Manually & None & Auto \\
\hline Range & $0.1 \sim 1.5 \mathrm{~km}$ & $0.1 \mathrm{~km}$ & $0.1 \mathrm{~km}$ \\
\hline $\begin{array}{c}\text { Battery Power } \\
\text { Profile }\end{array}$ & Years & Days & Hours \\
\hline $\begin{array}{c}\text { Standby current } \\
\text { (Amperes) }\end{array}$ & $3 \times 10^{-6}$ & $200 \times 10^{-6}$ & $20 \times 10^{-3}$ \\
\hline $\begin{array}{c}\text { Low power } \\
\text { consumption }\end{array}$ & Support & Not support & Not support \\
\hline
\end{tabular}

Finally, module costs of Bluetooth and $\mathrm{Wi}-\mathrm{Fi}$ are relatively high. In addition, the power consumption of Bluetooth and Wi-Fi compared to ZigBee is much higher. Although transmission data rate of Bluetooth and $\mathrm{Wi}-\mathrm{Fi}$ is higher than that of ZigBee, the $250 \mathrm{kbit} / \mathrm{s}$ data rate of ZigBee is enough for use in automatic monitor system for thermal processing, thus the ZigBee technology is selected.

The $2.4 \mathrm{GHz}$ Industrial Scientific Medical (ISM) band, which extends from 2400 to $2483.5 \mathrm{MHz}$ and is used worldwide, implements a half-sine-shaped Offset Quadrature Phase Shift Keying (O-QPSK) modulation format. Sixteen channels with data rate $250 \mathrm{kbit} / \mathrm{s}$ are available and, with minimum -96 dBm RF sensitivity required, the ideal transmission range is approximatively $500 \mathrm{~m}$. The ZigBee stack architecture, shown in Figure 3, is composed of a set of blocks called layers. Each layer performs a specific set of services for the layer above. Given the IEEE 802.15.4 specifications on PHY and MAC layers, the ZigBee Alliance provides the network layer and the framework for the application layer. The responsibilities of the ZigBee network layer include: mechanisms to join and leave a network, frame security, routing, path discovery, one-hop neighbours discovery, neighbour information storage. The ZigBee application layer consists of the application support sublayer, the application framework, the ZigBee device objects and the manufacturer-defined application objects. The responsibilities of the application support sublayer include: maintaining tables for binding (defined as the ability to match two devices together based on their services and their needs), and forwarding messages between bound devices. The responsibilities of the ZigBee device objects include: defining the role of the device within the network (e.g., WPAN coordinator or end device), initiating and/or responding to binding requests, establishing secure relationships between network devices, discovering devices in the network, and determining which application services they provide [5].

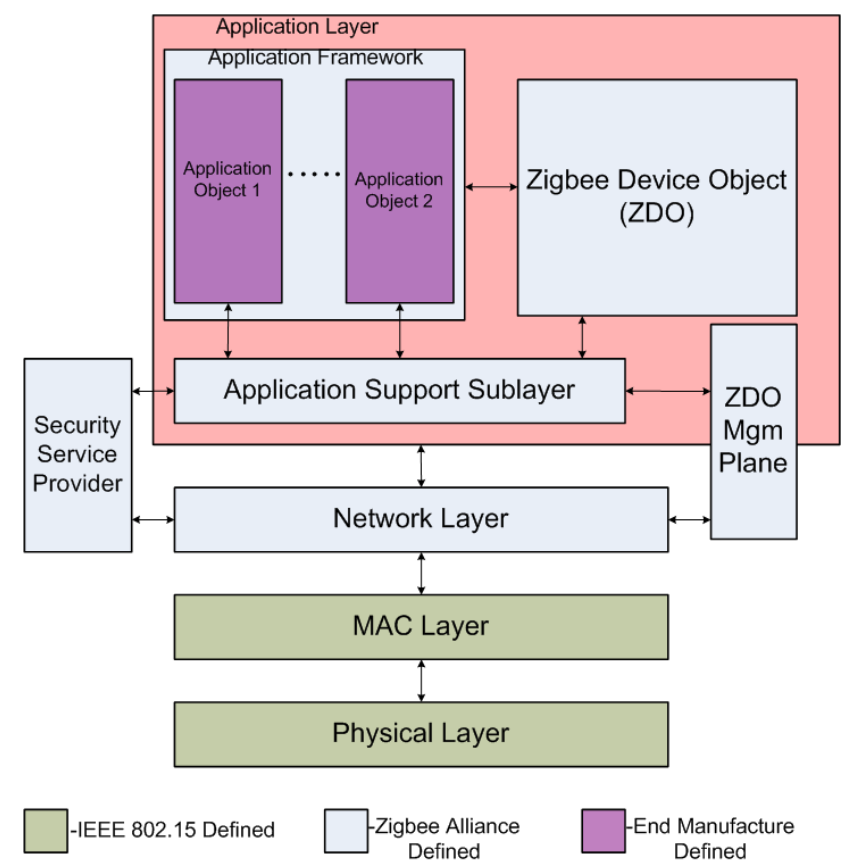

Figure 3: Communication layers

The MAC layer provides access control to a shared channel and reliable data delivery. IEEE 802.15.4 uses a protocol based on a CSMA/CA algorithm, which requires listening to the channel before transmitting to reduce the probability of collisions with other ongoing transmissions. The main functions performed by the MAC sublayer are: association and disassociation, security control, optional star network topology functions, such as beacon generation and Guaranteed Time Slots (GTSs) management, generation of ACK frames (if used), and, finally, application support for the two possible network topologies described in the standard. If two ZigBee devices $\mathrm{A}$ and $\mathrm{B}$ need to communicate with other and device A has two slots, which correspond to the two sensors on devices $\mathrm{B}$ respectively. If slot 1 on device A wishes to establish communication with the temperature sensor on device $\mathrm{B}$, it can request device $\mathrm{A}$ to establish a wireless communication channel to the device B, using either its IEEE 802.15.4 64-bit extend address or its 16-bit network address. The ZigBee specification defines a sub-level addressing mode-Endpoint, to help the system distinguish the multiple objects existing on one physical device. "Endpoint" is a kind of categorization, which virtually exists in the stack. Each ZigBee device can support up to 240 virtual objects (endpoint 0 is used for endpoint management). Each virtual object has its own property and can be independent from other objects. If the starter of the communication specifies which endpoint it is looking for, the ZigBee stack running on the destination ZigBee device can easily locate the target object. The concept of Endpoint in the ZigBee specification is useful, particular for wireless sensor networks. A terminal node is normally equipped with more than one sensor for executing multiple sensing tasks. 


\section{International Journal of Science and Research (IJSR) \\ ISSN (Online): 2319-7064}

Index Copernicus Value (2013): 6.14 | Impact Factor (2014): 5.611

\section{Design of the System}

\subsection{Hardware Structure}

Schematic diagram of the designed coordinator microprocessor system for thermal food process monitoring is given in Figure 4, and schematic diagram of the designed terminal node microprocessor system for thermal food process monitoring is given in Figure 5.

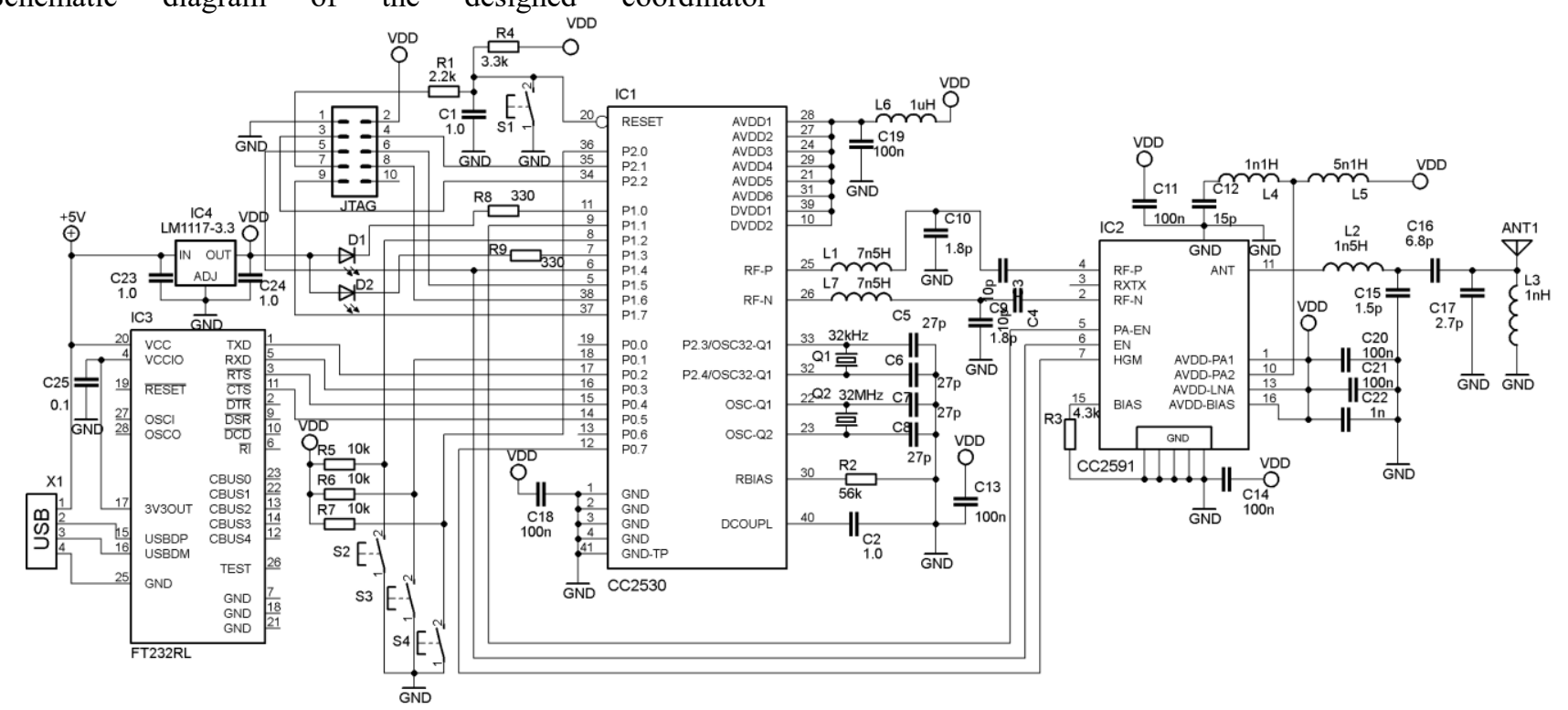

Figure 4: Schematic diagram of the coordinator microprocessor system

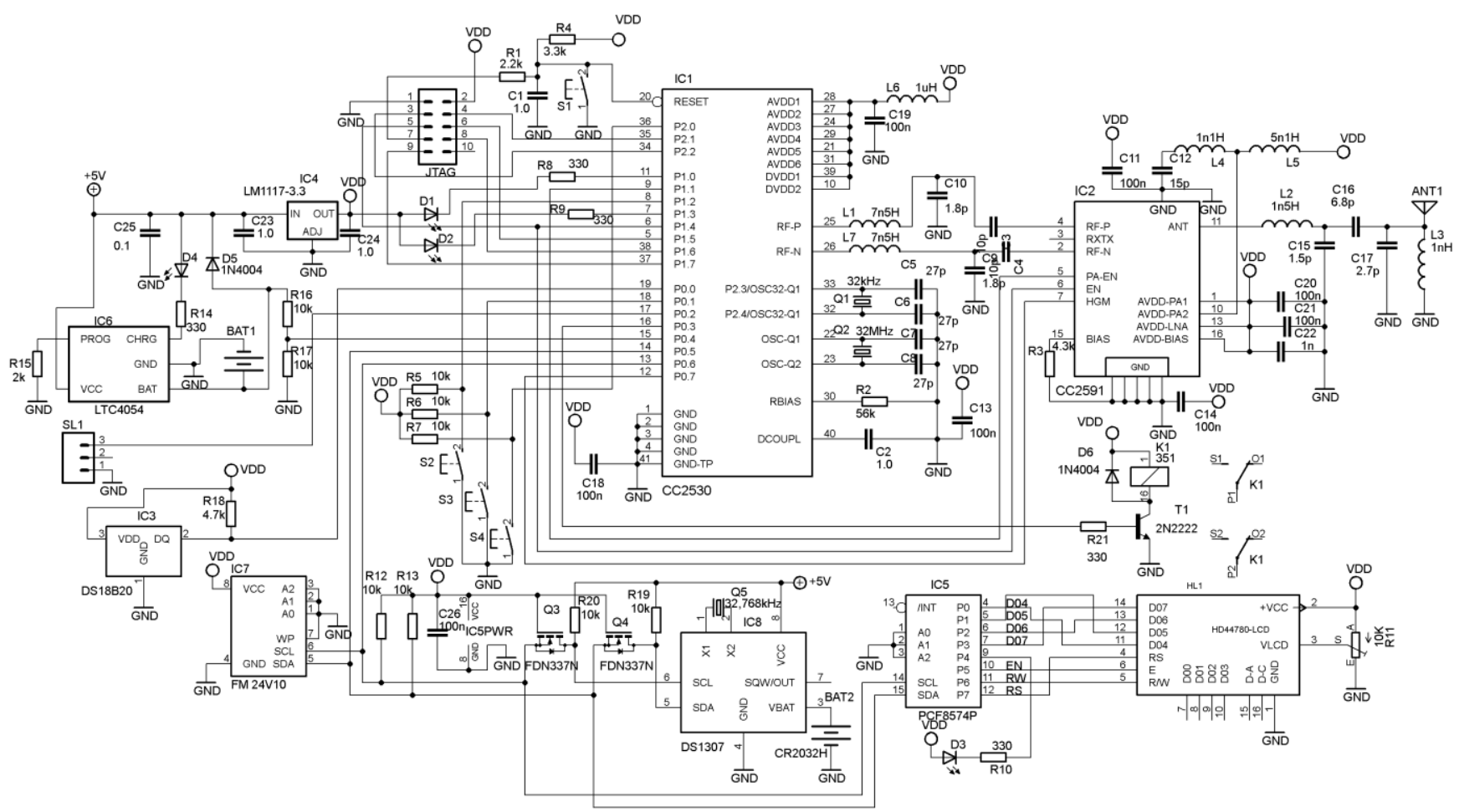

Figure 5: Schematic diagram of the terminal node microprocessor system

Hardware synthesis is base on System-on-Chip Solution (SoS) on embedded microcontroller CC2530 (IC1). The CC2530 combines the excellent performance of a leading RF transceiver with an industry-standard enhanced $8051 \mathrm{MCU}$, in-system programmable $256 \mathrm{~KB}$ flash memory, $8 \mathrm{~KB}$ RAM, The selected microcontroller also provided with the $A / D$ converter, multi-general timer, AES128 coprocessor, watchdog timer, sleep-mode timer with $32 \mathrm{kHz}$ crystals, reset circuit, power detection circuit and 21 programmable I/O pins. The chip support widespread Intel MCS-51 instruction set [4] and can use free development software. It has three different memory-access buses (SFR, DATA and CODE/XDATA) with single-cycle access to SFR, DATA, and the main SRAM. The memory arbiter is at the heart of the system, as it connects the CPU and DMA controller with the physical memories and all peripherals through the SFR bus. The memory arbiter has four memory access points, access of which can map to one of three physical memories: an 8-KB SRAM, flash memory, and XREG/SFR registers. It is responsible for performing arbitration and sequencing 


\section{International Journal of Science and Research (IJSR) \\ ISSN (Online): 2319-7064}

Index Copernicus Value (2013): 6.14 | Impact Factor (2014): 5.611

between simultaneous memories accesses to the same physical memory. The debug interface implements a proprietary two-wire serial interface that is used for in-circuit debugging. Through this debug interface, it is possible to perform an erasure of the entire flash memory, control which oscillators are enabled, stop and start execution of the user program, execute supplied instructions on the 8051 core, set code breakpoints, and single-step through instructions in the code. Using these techniques, it is possible to perform incircuit debugging and external flash programming elegantly. The I/O controller is responsible for all general-purpose $\mathrm{I} / \mathrm{O}$ pins. The CPU can configure whether peripheral modules control certain pins or whether they are under software control, and if so, whether each pin is configured as an input or output and if a pullup or pulldown resistor in the pad is connected. CPU interrupts can be enabled on each pin individually. Each peripheral that connects to the I/O pins can choose between two different $\mathrm{I} / \mathrm{O}$ pin locations to ensure flexibility in various applications. A versatile five-channel DMA controller is available in the system. Many of the hardware peripherals such as AES core, flash controller, USARTs, four timers, ADC interface achieve highly efficient operation by using the DMA controller for data transfers. The AES encryption/decryption core allows the user to encrypt and decrypt data using the AES algorithm with 128bit keys. The core is able to support the AES operations required by IEEE 802.15.4 MAC security, the ZigBee network layer, and the application layer. The CC2530 features an IEEE 802.15.4-compliant radio transceiver. The $\mathrm{RF}$ core controls the analog radio modules. In addition, it provides an interface between the MCU and the radio which makes it possible to issue commands, read status, and automates and sequence radio events. The radio also includes a packet-filtering and address-recognition module [6].

The microprocessor system is programmed by the JTAG connector with external CC Debugger for RF System-onChips of Texas Instrument.

The UART converter for communication with host personal computer is realized by FT232RL. This solution allows to use USB interface as bridge between host PC and the coordinator microprocessor system. The FT232RL builds a free Virtual Com Port (VCP) and eliminate the requirement for USB driver development. Data transfer rates from 300 baud to 3M baud. Special amplifier CC2591 for signal booster is used in the terminal node and the coordinator. The device is a range extender for all existing and future $2.4-\mathrm{GHz}$ low-power RF transceivers, transmitters, and System-on-Chip products from TI. The chip has low transmit current consumption under $100 \mathrm{~mA}$ and output power up to $22 \mathrm{dBm}$. In power down mode consumption is less than $100 \mathrm{nA}$. In receive mode current consumption is less than $3 \mathrm{~mA}$ [7]. The temperature senses via the latest waterproofed DS18B20 1wire digital temperature sensor from Maxim. It reports degrees with 9 to 12 -bit precision, from $-55^{\circ} \mathrm{C}$ to $125^{\circ} \mathrm{C}$ with accuracy $+/-0.5^{\circ} \mathrm{C}$. The sensor has a unique 64-Bit Serial number and it allows for a huge number of sensors to be used on one data bus. The DS1307 is used as real-time clock with calendar (RTC) as time logger of the food processing. The RTC is connected by $\mathrm{I}^{2} \mathrm{C}$ interface to the embedded microcontroller. The local LCD is base on HD44780 display interfaced by parallel to $\mathrm{I}^{2} \mathrm{C}$ converter PCF8574. The LCD is connected to the embedded controller via the $\mathrm{I}^{2} \mathrm{C}$ bus. The FRAM $24 \mathrm{~V} 10$ is used to store data of thermal processing. The LTC4054 provides a buffer against power supply interruptions. The Relay K1 controls the start and stop of the thermal process. The button $\mathrm{S} 1$ resets the systems. The others buttons are for local configuration of the coordinator and terminal node.

\subsection{Software Structure}

Software design is based on the ZigBee free protocol stack of Texas Instruments (TI) for x51 core and C programming Embedded Workbench as the software platform. It includes the software design of coordinator and terminal nodes. The program flow chart of ZigBee coordinator is showed in Figure 5.

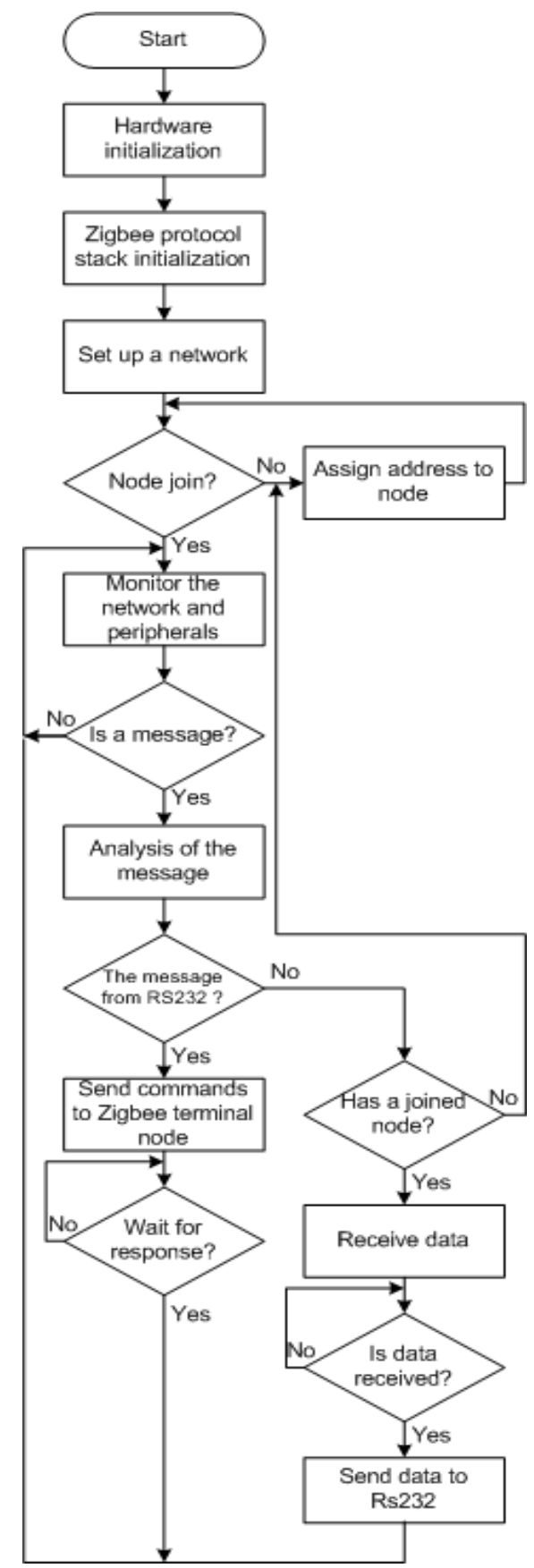

Figure 5: Coordinator Program flow chart 


\section{International Journal of Science and Research (IJSR) \\ ISSN (Online): 2319-7064 \\ Index Copernicus Value (2013): 6.14 | Impact Factor (2014): 5.611}

Visualization of data is made by Visual Basic platform and includes serial communication program, data and command console. The $\mathrm{Z}$ - Stack of TI was adopted for specific tasks of thermal processing. Z-Stack is constructed by the idea of the operating system, using event round robin.

The program flow chart of ZigBee terminal node is showed in Figure 6.

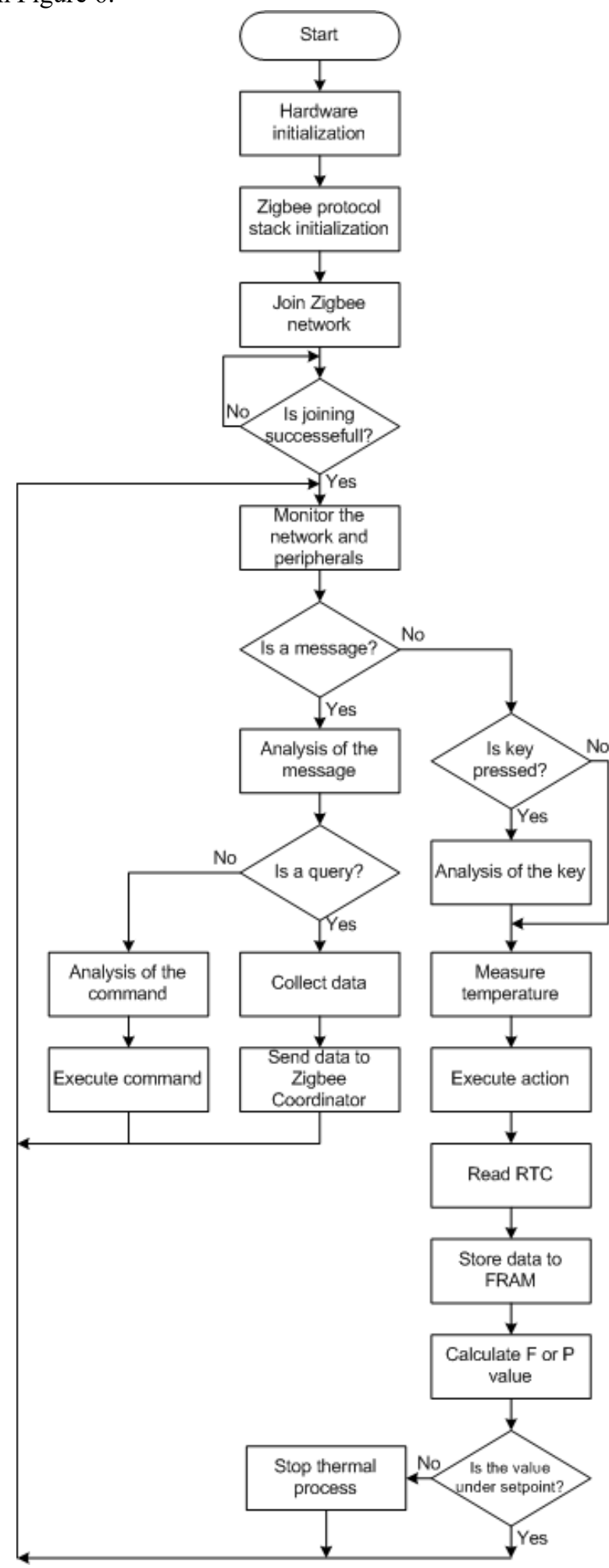

Figure 6: Terminal node Program flow chart

When no events happened, the system enters a low-power mode. When an event occurs, the system is woke up and begins to handle the event, then the system enters a low power mode. If several events are occurred at the same time, determining the priorities, then processing the event one by one. This software framework can greatly reduce the power consumption of the system. Every part of microprocessor system has different tasks. The coordinator is the core of Zigbee network, which is responsible for network establishment, address assignment, information management, data receiving and commands transmission to nodes. In addition, it communicates with the PC through RS232 serial port. The main function of terminal nodes is to join the existing wireless sensor network, gather temperature data, calculate lethal $\mathrm{F}$ value, send data to coordinator and execute relative commands to control different thermal processes.

\subsection{System analysis}

The prototype microprocessor system is shown in Figure 7.

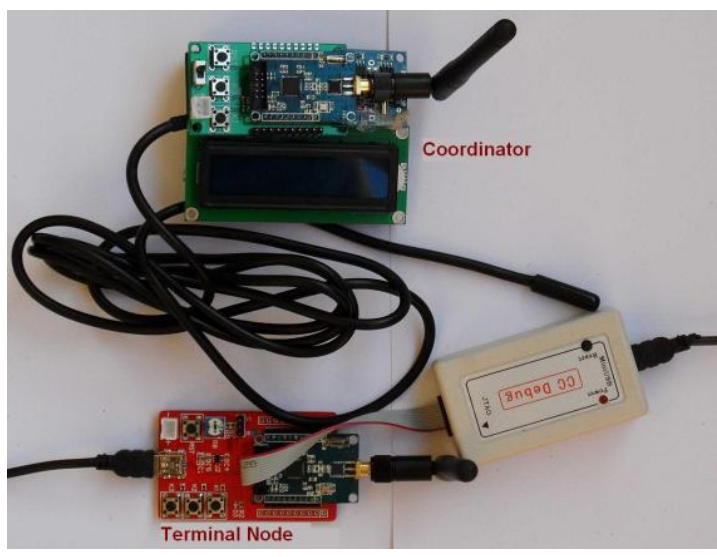

Figure 7: Prototype microprocessor system

The system test is made in real thermal food process. The experimental data of process is visualized in chart mode by GUI application as is shown in Figure 8.

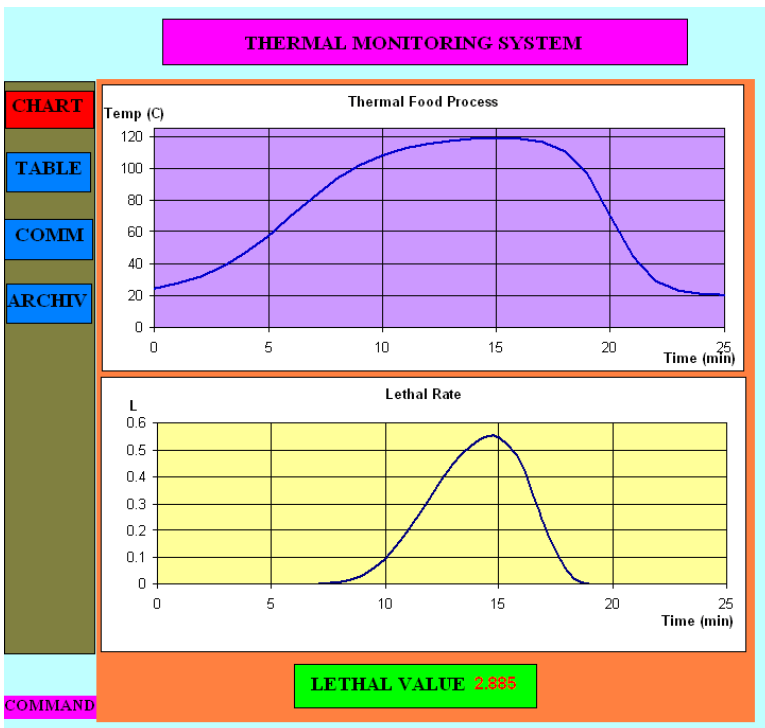

Figure 8: Visualized experimental data in chart mode

The experimental data of process is visualized in table mode by GUI application as is shown in Figure 9. 


\section{International Journal of Science and Research (IJSR) \\ ISSN (Online): 2319-7064}

Index Copernicus Value (2013): 6.14 | Impact Factor (2014): 5.611

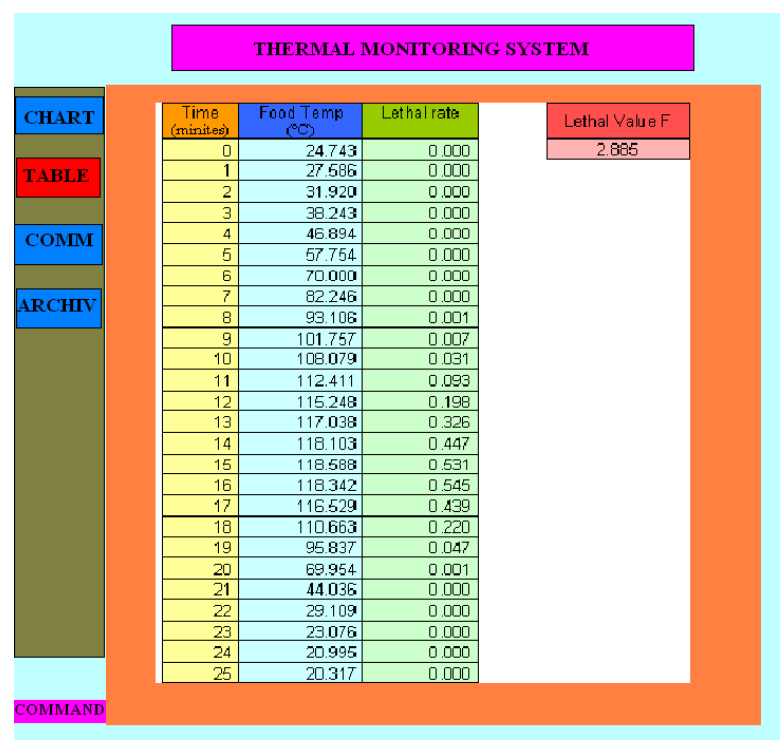

Figure 9: Visualized experimental data in table mode

In every moment the operator monitors thermal process and can send command to terminal node. If operator sends command archive the all thermal process data from terminal node are archived on local host computer. The system was tested for network range terminal node discovery. In industrial environment, the experiment shown, that terminal node has stable network connection with range more than $500 \mathrm{~m}$.

\section{Conclusion}

An innovative microprocessor system which can achieve temperature remote monitoring of thermal food processing is proposed. The thermal food monitoring system based on ZigBee technology is a new solution that used to meet the demand of high intelligent automation. The presented microprocessor-based system is low priced and ensures implementation of various control algorithms for food processing monitoring. The details of hardware and software design in thermal food monitoring system are given, which include the design of coordinator, terminal nodes. Operating results of the device are very good so far, no defects and failures. Experimental results of actual food processing show that the proposed system not only overcome the lack of traditional wire network, but also can monitor the thermal food processing efficiently. The microprocessor system with five nodes and one coordinator has low price below 150 EUR and high reliability because it is built on the quality embedded microcontroller CC2530. The use of the amplifier CC2591 as signal booster allows extended communication in industrial environment with range more than $500 \mathrm{~m}$. The proposed system has significance advance over classic systems for thermal food monitoring with wire connection.

\section{References}

[1] Barbosa-Canovas G., Thermal Processing of Packaged Foods, second edition, Springer Science, 2007.

[2] Featherstone S., A Complete Course in Canning and Related Processes, Fourteenth Edition, Elsevier Ltd, 2015.
[3] IEEE Computer Society, IEEE Standard 802.15.4-2003: Part 15.4: Wireless Medium Access Control (MAC) and Physical Layer (PHY) Specifications for Low-Rate Wireless Personal Area Networks (LR-WPANs), Institute of Electrical and Electronics Engineers, 2003.

[4] Mazidi M., R. McKinlay, J. Mazidi, The 8051 Microcontroller: A Systems Approach, Pearson Education Ltd., 2014.

[5] Shuang-Hua Yang, Wireless Sensor Networks: Principles, Design and Applications, Springer-Verlag, 2014.

[6] Texas Instruments Incorporated, CC2530F256 A True System-on-Chip Solution for 2.4-GHz IEEE 802.15.4 and ZigBee Applications, SWRS081B, 2011.

[7] Texas Instruments Incorporated, CC2591 2.4-GHz RF Front End, SWRS070B, 2014.

\section{Author Profile}

Dr. Krassimir Kolev received his Ph.D. in Communications and Computing Technology from the University of Food Technologies - Plovdiv (UFT), Bulgaria. He is the chief assistant professor of Computer Systems, Complexes and Networks in the department of Computer Systems and Technologies of the UFT. Dr. Kolev has over 20 years of teaching experience of Computer Systems. His current research projects are in the areas of microprocessor systems, programmable controllers, embedded systems and image processing. 